\title{
DIÓXIDO DE CLORO Y DERIVADOS DEL CLORO PARA PREVENIR O TRATAR LA COVID-19: REVISIÓN SISTEMÁTICA
}

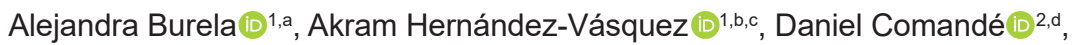 \\ Verónica Peralta (1) ${ }^{1, b, e}$, Fabian Fiestas (1) ${ }^{1, b}$ \\ 1 Instituto de Evaluación de Tecnologías en Salud e Investigación - IETSI, EsSalud, Lima, Perú. \\ 2 Instituto de Efectividad Clínica y Sanitaria (IECS), Buenos Aires, Argentina. \\ a Bióloga; ${ }^{b}$ médico/a cirujano/a; ${ }^{\mathrm{c}}$ magíster en Gestión y Políticas Públicas; ${ }^{\mathrm{d}}$ licenciado en Bibliotecología y Ciencia de la \\ Información; ${ }^{\mathrm{e}}$ especialista en Gestión en Salud.
}

\section{RESUMEN}

Objetivos: Realizar una revisión sistemática acerca de la efectividad y seguridad del uso de dióxido de cloro y derivados del cloro, en la prevención o el tratamiento de la COVID-19. Materiales y métodos: Se siguieron las pautas internacionales de elaboración de revisiones sistemáticas de PRISMA y el Manual Cochrane para revisiones sistemáticas de intervenciones. La estrategia de búsqueda la desarrolló un bibliotecario y la revisaron dos de los autores. Se complementó la búsqueda electrónica con una búsqueda manual. Se incluyeron ensayos clínicos aleatorizados, estudios cuasiexperimentales, estudios de cohorte, estudios de casos y controles, estudios de corte transversal y reportes de casos; y se excluyeron estudios in vitro o realizados en animales. Dos revisores, de forma independiente, seleccionaron los estudios según los criterios de elegibilidad definidos, usando el aplicativo web Rayyan, en caso de discordancia se hizo partícipe a un tercer revisor. El protocolo de la revisión sistemática se registró en PROSPERO (CRD42020200641). Resultados: No se identificó ningún estudio publicado ni en proceso de publicación que haya evaluado el uso del dióxido de cloro o derivados del cloro, administrado por vía inhalatoria, oral o parenteral en humanos, como agente preventivo o terapéutico de la COVID-19 o en infecciones por otros coronavirus. Solo se identificó el registro de un único estudio catalogado como observacional que hasta ahora no tiene resultados. Conclusiones: A la fecha, no existe evidencia científica que apoye el uso del dióxido de cloro o derivados del cloro para prevenir o tratar la COVID-19.

Palabras clave: Dióxido de Cloro; Compuestos de Cloro; Prevención y Control; Síndrome Respiratorio Agudo Grave Coronavirus 2; COVID-19; Infección por SARS-CoV-2 (Fuente: DeCS BIREME).

\section{CHLORINE DIOXIDE AND CHLORINE DERIVATIVES FOR THE PREVENTION OR TREATMENT OF COVID-19: A SYSTEMATIC REVIEW}

Citar como: Burela A, HernándezVásquez A, Comandé D, Peralta V, Fiestas F. Dióxido de cloro y derivados del cloro para prevenir o tratar la COVID-19: revisión sistemática. Rev Peru Med Exp Salud Publica. 2020;37(4):605-10. doi: https://doi. org/10.17843/rpmesp.2020.374.6330.

Correspondencia: Verónica Victoria Peralta Aguilar; Av. Arenales 1400, Jesús María, Lima, Perú; veronica. peralta@essalud.gob.pe

Recibido: $11 / 08 / 2020$ Aprobado: 02/09/2020 En línea: 07/09/2020

\section{ABSTRACT}

Objectives: To systematically review the effectiveness and safety of chlorine dioxide solution and chlorine derivatives used in the prevention or treatment of COVID-19. Methods: This review adheres to the Preferred Reporting Items for Systematic Reviews and Meta-analysis (PRISMA) and follows the guidelines provided in the Cochrane Handbook for Systematic Reviews of Interventions. A librarian developed and executed the search strategy; it was further reviewed by two of the authors and complemented by manual search. Randomized clinical trials, quasi-experimental studies, cohort studies, case-control studies, cross-sectional studies, and case reports were included; in vitro or animal studies were excluded. Abstract and full-text screening according to pre-defined eligibility criteria were performed by two reviewers independently using web application Rayyan QCRI. Disagreements on study selection were resolved by a third reviewer. The systematic review protocol was registered in PROSPERO (CRD42020200641). Results: Neither published nor pre-print studies evaluating the use of chlorine dioxide or derivatives on SARS-CoV-2 infection were identified. The only finding was an unpublished observational study registry which has no results released yet. Conclusions: To date, there are no scientific evidence to uphold the use of chlorine dioxide or derivatives as preventive or therapeutic agents against COVID-19.

Keywords: Chlorine Dioxide; Chlorine Compounds; Prevention and Control; Severe Acute Respiratory Syndrome Coronavirus 2; COVID-19; SARS-CoV-2 infection (Source: MeSH NLM). 


\section{INTRODUCCIÓN}

En marzo del 2020, la Organización Mundial de la Salud (OMS) declaró a la enfermedad por coronavirus 2019 (COVID-19) como una pandemia. Esta enfermedad se reportó por primera vez, en diciembre del 2019, en la ciudad china de Wuhan, y se identificó posteriormente un nuevo tipo de coronavirus como el agente causante (SARS-CoV-2) ${ }^{(1)}$. Al 9 de agosto del presente año, en todo el mundo se han reportado más de 19 millones de casos confirmados de COVID-19 y en el Perú se han confirmado 471012 casos y 20844 muertes ocasionadas por esta enfermedad ${ }^{(2)}$.

La principal vía de transmisión es de persona a persona, a través de gotículas expelidas por una persona infectada, al momento de estornudar, toser e incluso hablar, y de manera indirecta mediante fómites. Adicionalmente, en circunstancias y lugares específicos que lleven a la producción de aerosoles también podría darse transmisión por vía aérea ${ }^{(3)}$. En los casos leves a moderados los síntomas son similares a un resfrío común y puede presentarse o no una neumonía leve; algunas veces se presentan síntomas neurológicos y gastrointestinales, usualmente sin necesidad de hospitalización. En los casos graves se presenta disnea e hipoxia, con un compromiso de más del 50\% del tejido pulmonar, casos en los que se requiere oxigenoterapia o ventilación mecánica. Los pacientes críticos presentan un compromiso multiorgánico, así como una intensa respuesta inflamatoria, pueden llegar a desarrollar sepsis y un síndrome de disfunción multiorgánica que ocasione la muerte ${ }^{(4-6)}$.

Aún no se dispone de agentes preventivos ni de tratamientos curativos contra el SARS-CoV-2 ${ }^{(7,8)}$, debido a que no existe evidencia científica que apruebe el uso de los medicamentos evaluados. Así, en la actualidad solo se tienen tratamientos sintomáticos o de control de la enfermedad. Debido a este vacío terapéutico, en todo el mundo se utilizan distintos agentes que no cuentan con evidencia científica ${ }^{(9)}$. Sumado a esto, en el mercado peruano se ofrecen algunos productos que, además de no tener sustento científico para su uso contra la COVID-19, carecen de autorización sanitaria para ser utilizados en humanos como tratamiento médico ${ }^{(10)}$. No obstante, los distribuidores y los fabricantes de dichos productos aseguran su eficacia y seguridad contra la COVID-19, como agentes preventivos, curativos y de control de la enfermedad ${ }^{(11)}$.

Los productos a los que se hace referencia previamente son el dióxido de cloro, el clorito de sodio y otros derivados del cloro. Comercialmente al dióxido de cloro se le conoce como CDS (siglas de chlorine dioxide solution) publicitado como un derivado del compuesto inicialmente vendido bajo el nombre de SMM (sustancia mineral milagrosa) o MMS (siglas de mineral miracle solution) ${ }^{(12)}$, el cual contiene clorito de sodio. Al mezclarse en agua con un ácido, preparación indicada por sus distribuidores, el clorito de sodio se convierte

\section{MENSAJES CLAVE}

\begin{abstract}
Motivación para realizar el estudio: Cada vez son más las personas que, a falta de un medicamento efectivo para prevenir o curar la COVID-19, ingieren dióxido de cloro o derivados del cloro, compuestos químicos no autorizados para consumo humano.
\end{abstract}

Principales hallazgos: A la fecha no existe evidencia científica que respalde el uso del dióxido de cloro ni de los derivados del cloro para prevenir o tratar la COVID-19.

Implicancias: Ante la falta de evidencia, estos compuestos químicos no pueden considerarse eficaces ni seguros. Dicho esto, la comunidad científica y médica ha manifestado su preocupación por los daños que puede causar su consumo en las personas.

en ácido cloroso para finalmente convertirse en dióxido de cloro. Por otro lado, el CDS es el gas de dióxido de cloro en solución. Tanto el dióxido de cloro como otros derivados del cloro (ej., clorito de sodio, hipoclorito de sodio, etc.) son utilizados como agentes desinfectantes en distintos procesos industriales, debido a su fuerte poder oxidante ${ }^{(13,14)}$. Este efecto oxidante termina por desnaturalizar compuestos orgánicos, no obstante, su efecto no es específico para un tipo de organismo en particular.

En este escenario, se requiere recabar evidencia científica para contrastar las aseveraciones difundidas y la hipótesis planteada a favor del uso de CDS o SMM como un agente preventivo y como tratamiento curativo o de control de la COVID-19 ${ }^{(15)}$. Dicho esto, el presente estudio tuvo como objetivo revisar sistemáticamente la eficacia y seguridad del uso de dióxido de cloro y derivados del cloro, en la prevención o tratamiento de la COVID-19.

\section{MATERIALES Y MÉTODOS}

Para realizar la presente revisión sistemática, se siguió la declaración de ítems de referencia para publicar revisiones sistemáticas y metaanálisis (PRISMA, por sus siglas en inglés) ${ }^{(16)}$ y el Manual Cochrane para revisiones sistemáticas de intervenciones ${ }^{(17)}$. El protocolo de la revisión sistemática se registró en PROSPERO con el número de referencia CRD42020200641 (https://www.crd. york.ac.uk/prospero/display_record.php?RecordID=200641).

\section{Criterios de elegibilidad}

Se incluyeron ensayos clínicos aleatorizados, estudios cuasiexperimentales, estudios de cohorte, estudios de casos y controles, estudios de corte transversal, y reporte de casos que evaluaron dióxido de cloro y derivados del cloro para 
prevenir o tratar la COVID-19 en personas de todas las edades. No se aplicaron restricciones de idiomas ni de estado de publicación. Asimismo, se consideró incluir estudios en otras infecciones por coronavirus (ej., MERS-CoV, SARS$\mathrm{CoV}$, etc.) en caso de no encontrar estudios realizados en infecciones por SARS-CoV-2. Se excluyeron estudios in vitro o realizados en animales.

\section{Estrategias de búsqueda}

Se realizó una búsqueda electrónica sistemática en las bases de datos PubMed, Embase (Excerpta Medica Database), CINAHL (Cumulative Index to Nursing and Allied Health Literature), Cochrane Library, Web of Science, LILACS (Literatura Latinoamericana y del Caribe en Ciencias de la Salud), y SciELO (Scientific Electronic Library Online) de artículos publicados hasta el 24 de julio de 2020. Se diseñó inicialmente una estrategia de búsqueda en PubMed adaptada a las otras bases de datos combinando los siguientes términos con sinónimos y otros términos de descriptores médicos: «coronavirus», «SARS-CoV-2», y «Chlorine».

Un bibliotecario (DC) desarrolló las estrategias de búsqueda que fueron validadas por dos de los autores ( $\mathrm{AB}$ y $\mathrm{AHV}$ ). Los términos de búsqueda utilizados para las bases de datos se detallan en el Anexo 1 del material suplementario. La búsqueda electrónica se complementó con búsquedas manuales en las listas de referencias de artículos relevantes para identificar posibles estudios que no se hayan encontrado en la búsqueda electrónica (estrategia tipo bola de nieve), en Google Académico (diez primeras páginas de resultados) y en repositorios de preimpresiones (medRxiv y bioRxiv) mediante una combinación de los siguientes términos: «coronavirus», «SARS-CoV-2», y «Chlorine». Adicionalmente, se revisaron registros de ensayos clínicos en progreso o con datos no publicados en el Registro de Ensayos Clínicos de China (ChiCTR, por sus siglas en inglés), en el Registro de Ensayos Clínicos de los Países Bajos (NTR, por sus siglas en inglés), ClinicalTrials.gov, y en el registro del Número Internacional de Ensayo Clínico Controlado Aleatorizado Normalizado (ISRCTN, por sus siglas en inglés). La búsqueda se realizó sin restricciones de diseño de estudio, estado de publicación, fecha o idioma de publicación.

\section{Selección de estudios}

En una primera etapa, los resultados de la búsqueda electrónica y manual se importaron al software de gestión de referencias EndNote X9 (licencia 3061914708). Luego, se eliminaron todos los registros duplicados siguiendo la metodología descrita por Bramer et al. ${ }^{(18)}$. Se evaluó si los registros identificados cumplían con los criterios de inclusión y se excluyó de la revisión a los que no cumplían con dichos criterios. Dos revisores de forma independiente (AB y AHV) participaron en la evaluación de la elegibilidad de todos los registros mediante el aplicativo web Rayyan (https://rayyan. qcri.org/ ${ }^{(19)}$. Cualquier desacuerdo se resolvió entre los dos revisores y en caso de no poder tomar una decisión, un tercer revisor participó en la discusión (VP).

\section{Extracción y síntesis de resultados}

Se planificó el reporte de cualquier tipo de desenlace del uso del dióxido de cloro y derivados del cloro para prevenir o tratar la COVID-19, tales como la tasa de curación, el tiempo de resolución, la reducción en la gravedad de la enfermedad, el periodo de hospitalización, la tasa de mortalidad, los eventos adversos, entre otros, además de extraer las características generales de cada estudio y evaluar la calidad de cada uno de ellos según el tipo de estudio. Sin embargo, no se encontró ningún estudio que cumpla con los criterios de inclusión para realizar estos procesos.

\section{Consideraciones éticas}

No se solicitó la aprobación del estudio por parte de un comité de ética institucional por tratarse de una revisión de bases de datos bibliográficas.

\section{RESULTADOS}

La búsqueda en las bases de datos y otras fuentes incluyó 101 registros luego de eliminar duplicados, de los cuales no se identificó ningún estudio publicado o en proceso de publicación, como preimpresión, que haya evaluado el uso del dióxido de cloro y de derivados del cloro para prevenir o tratar la COVID-19 o infecciones por otros coronavirus (Figura 1).

Adicionalmente, producto de la revisión de registros de ensayos clínicos, se identificó un único registro en ClinicalTrials.gov (NCT04343742) titulado «Determination of the Effectiveness of Oral Chlorine Dioxide in the Treatment of COVID 19», inscrito como estudio observacional que tiene planificado incluir a 20 participantes colombianos para evaluar la eficacia del dióxido de cloro oral en el tratamiento de pacientes con infección por COVID-19 ${ }^{(20)}$.

\section{DISCUSIÓN}

Según los resultados de la revisión sistemática realizada, no existe ningún tipo de evidencia científica publicada ni en proceso de publicación que haya evaluado el uso del dióxido de cloro o derivados del cloro como agente preventivo o terapéutico contra la COVID-19 administrado por vía inhalatoria, oral o parenteral. Solo se identificó el registro de un estudio en ClinicalTrials.gov catalogado como observacional que a la fecha no cuenta con resultados. Asimismo, debido a lo reciente de este tipo de coronavirus, se amplió la búsqueda incluyendo otros tipos de coronavirus, para los cuales tampoco se identificó ningún tipo de evidencia. Esta 


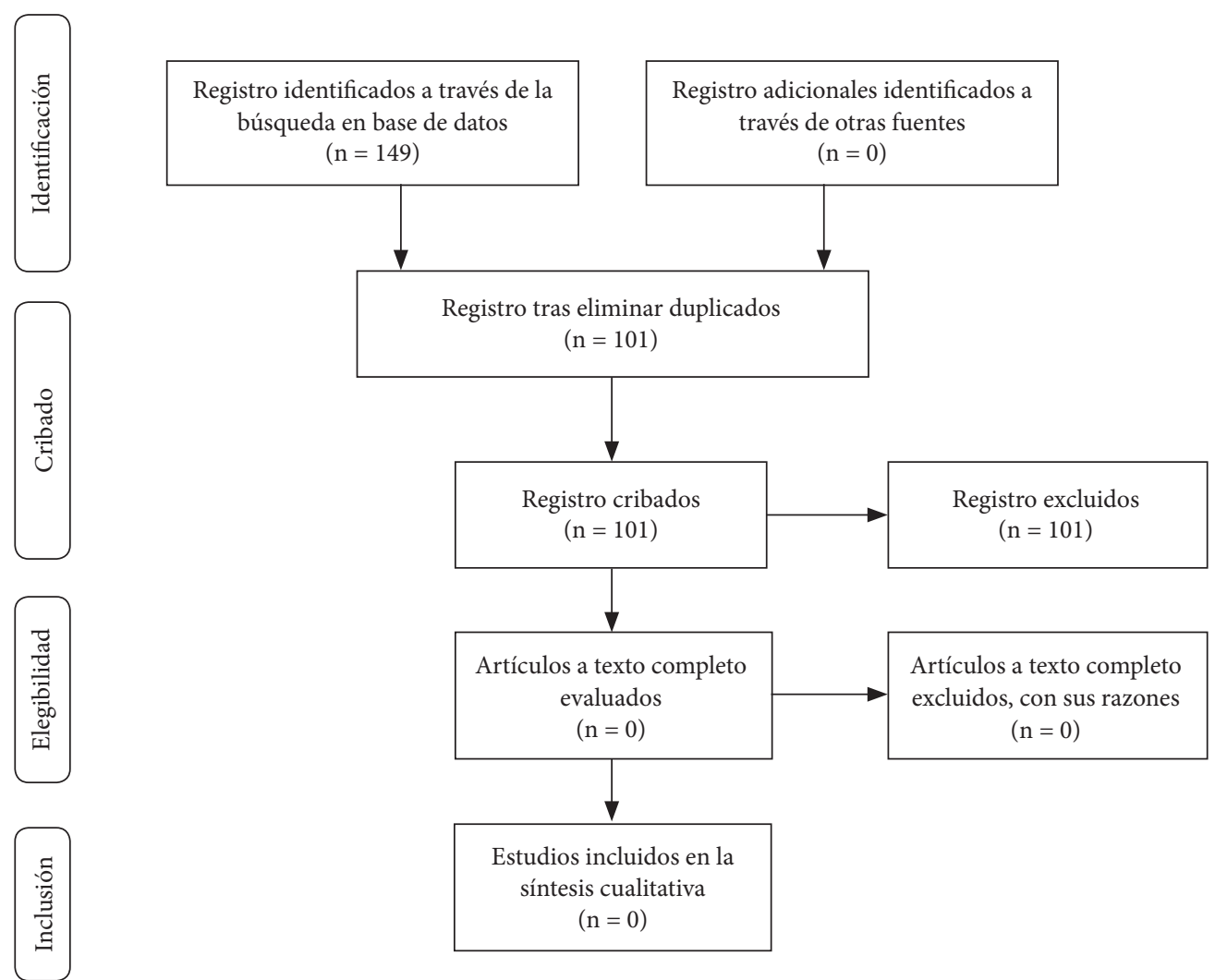

Figura 1. Flujo de la selección de estudios según la declaración PRISMA.

falta de evidencia también ha sido reportada por un documento técnico de síntesis de evidencia del Instituto Nacional de Salud del Perú ${ }^{(21)}$.

Previo al surgimiento de la pandemia por COVID-19, ocasionada por el virus SARS-CoV-2, el uso de productos derivados del cloro, ya eran comercializados en algunos países europeos y en los Estados Unidos. Específicamente el clorito de sodio, bajo el nombre de SMM o MMS, cuyos distribuidores alegaban que, luego de mezclarse con un ácido, pasaba a tener un efecto antimicrobiano, antiviral y antibacterial, lo que constituía un tratamiento para distintas enfermedades no relacionadas, como trastorno del espectro autista, neoplasias, hepatitis y VIH/SIDA ${ }^{(22)}$. Existen varios comunicados antes y después de la actual pandemia por COVID-19 emitidos por entidades regulatorias, cuya finalidad es denunciar y exigir el retiro de este producto del mercado dentro de esos países ${ }^{(23-25)}$.

No obstante, dentro de las Américas el dióxido de cloro y el clorito de sodio han seguido siendo ofertados con especial relevancia a raíz de la pandemia por SARS-CoV-2; se promociona su uso no solo como tratamiento para las enfermedades previamente mencionadas, sino también como agente preventivo y de tratamiento para la infección por SARSCoV-2. La Organización Panamericana de la Salud (OPS) ${ }^{(26)}$ no recomienda el uso de dióxido de cloro ni del clorito de sodio por vía oral o parenteral en pacientes con sospecha o con diagnóstico de COVID-19. Menciona, además, que no debe utilizarse en ningún otro tipo de enfermedad, puesto que no existe evidencia acerca de su eficacia; por el contrario, la ingesta o inhalación de dichos productos generaría graves efectos adversos. La Administración de Alimentos y Medicamentos de los Estados Unidos (FDA, por sus siglas en inglés) ha recibido notificaciones de eventos adversos ocasionados por estos productos, como falla respiratoria por metahemoglobinemia, arritmia cardiaca a causa de la prolongación del intervalo QT, hipotensión por desequilibrio hidroelectrolíticos, insuficiencia hepática aguda, anemia hemolítica, vómitos y diarrea aguda severa ${ }^{(24,27)}$.

El concepto de "primero no hacer daño» es un principio fundamental en la salud y en la vida. Como se mencionó previamente, el dióxido de cloro y los derivados del cloro se utilizan de manera rutinaria en procesos industriales, por ejemplo, en la potabilización del agua para consumo humano. Debe tenerse muy en cuenta que los efectos en la salud que pueda ocasionar una sustancia siempre dependerá de la dosis, la duración y la forma de la exposición, la presencia de otras sustancias, las características y los hábitos personales y el estado de salud de cada persona ${ }^{(13)}$. Es por ello, que existen límites máximos permitidos de la cantidad de dióxido de cloro y clorito por volumen de agua en el proceso de potabilización. No obstante, los productos ofertados para prevenir y tratar la COVID-19, además de no contar con evidencia científica so- 
bre su eficacia, carecen de registro sanitario, con lo cual no es posible estandarizar una dosis máxima que al menos permita asegurar que dicha sustancia pueda ser inocua al encontrarse dentro de los límites establecidos para prevenir eventos adversos. De hecho, en los países donde se ha comercializado el dióxido de cloro o el clorito de sodio como agentes terapéuticos, se observó que las concentraciones de estos productos superan los límites máximos permitidos en el agua potable ${ }^{(23)}$. Es decir, estos productos comercializados como agentes preventivos o tratamientos por vía oral o parenteral no solo no cuentan con evidencia científica clínica que haya demostrado ser de beneficio para algún tipo de enfermedad, sino que, además, podrían ocasionar daños serios.

En el Perú, la Dirección General de Medicamentos, Insumos y Drogas también se ha pronunciado en contra del uso de estas sustancias para tratar alguna enfermedad, como la COVID-19, y advierte que la promoción y comercialización de este tipo de productos es ilegal y ha informado que su consumo podría ocasionar daños potencialmente mortales ${ }^{(10)}$.

Como se ha podido mostrar en los resultados de esta revisión sistemática, cuya finalidad fue tratar de identificar algún estudio científico publicado o en proceso de publicación con respecto a la eficacia y seguridad de la administración por vía inhalatoria, oral o parenteral del dióxido de cloro, clorito de sodio o derivados del cloro, ninguna entidad académica, de investigación o de salud del mundo ha visto razonable estudiar los potenciales efectos preventivos o terapéuticos de esta sustancia, atribuidos por sus distribuidores, en el contexto de COVID-19, a pesar de que a la fecha existe un vacío terapéutico.

Difundir argumentos tratando de usar un aparente lenguaje científico puede ocasionar que el consumidor se confunda, por lo tanto, es necesario entender el mecanismo de acción de los agentes oxidantes, como el dióxido de cloro, el clorito de sodio, el hipoclorito de sodio, entre otros. El cual recae en su poder oxidativo, es decir, que estas sustancias sirven como desinfectantes puesto que pueden oxidar otros compuestos a través de una reacción de óxido-reducción (también conocida como REDOX), en la cual el agente oxidante se reduce al ganar electrones, mientras que el reductor se oxida al perder electrones ${ }^{(28)}$. Este efecto oxidante termina por desnaturalizar los compuestos orgánicos ${ }^{(14,29)}$. Sin embargo, su efecto no es específico para un organismo en particular, ya que todos los organismos vivos estamos compuestos por moléculas orgánicas, por lo tanto, nuestras células, al igual que otros microorganismos, también estarían siendo afectadas ${ }^{(30)}$.

\section{REFERENCIAS BIBLIOGRÁFICAS}

1. Coronavirus: the first three months as it happened. Nature. 2020;10.1038/ d41586-020-00154-w. doi: 10.1038/d41586-020-00154-w.

2. Brote de enfermedad por el Coronavirus (COVID-19) [Internet]. OPS; 2020 [citado el 10 de agosto de 2020]. Disponible en: https:// www.paho.org/es/temas/coronavirus/brote-enfermedad-por-coronavirus-covid-19.
El uso de productos que no han probado ser eficaces y seguros contra la COVID-19 no solo supone un potencial peligro en la salud de quienes los utilicen, sino también de toda la población. Al generarse una falsa sensación de seguridad, por el supuesto efecto beneficioso no probado del producto, puede incurrirse en el abandono de las medidas de prevención y control frente a la COVID-19 que sí han probado ser eficaces, como el uso de mascarillas, el distanciamiento social, la higiene de manos y la etiqueta respiratoria ${ }^{(3,31)}$. Por lo tanto, es un derecho colectivo ser responsable con respecto a las medidas de prevención y control que cada individuo utilice.

Aunque se realizó una revisión exhaustiva de la literatura, podría haber fuentes adicionales en otras bases de datos bibliográficas o repositorios regionales. Sin embargo, el presente estudio incluyó las bases de datos más grandes e importantes del área biomédica, literatura gris y repositorio de preimpresiones, sin aplicar ninguna restricción a la inclusión de estudios según diseño, estado de publicación, fecha o idioma de publicación, siempre y cuando estos fueran realizados en humanos.

Se concluye que a la fecha, no existe evidencia científica que apoye el uso de dióxido de cloro o derivados del cloro para prevenir o tratar la COVID-19. Esto posiblemente se debe a que no existiría plausibilidad biológica ni indicios clínicos preliminares que permitan formular hipótesis coherentes para respaldar el desarrollo de estudios clínicos sobre el uso del dióxido de cloro o derivados del cloro como agentes terapéuticos o de prevención; además, a la preocupación de la comunidad médica por la toxicidad de dichos productos.

Contribuciones de autoría: FF, AB y AHV concibieron la idea de la revisión. $\mathrm{DC}$ realizó las búsquedas y las validó con $\mathrm{AB}$ y $\mathrm{AHV}$. $\mathrm{AB}$ y AHV se encargaron de la selección de estudios y redactaron el primer borrador de la revisión. VP coordinó la elaboración de la revisión. Todos los autores revisaron críticamente el manuscrito, aprobaron la versión final y asumen responsabilidad frente a todos los aspectos del artículo.

Financiamiento: Este estudio fue financiado por el Instituto de Evaluación de Tecnologías en Salud e Investigación (IETSI), EsSalud, Perú.

Conflictos de interés: VP y FF son funcionarios del IETSI. Asimismo, AB y AHV son consultores externos de dicho instituto. AHV es miembro del comité editor de la Revista Peruana de Medicina Experimental y Salud Pública, y no tuvo participación en ninguna etapa del proceso editorial posterior al envío del presente artículo. DC declara no tener conflictos de interés.

Material suplementario: Disponible en la versión electrónica de la RPMESP. 
5. Wu Z, McGoogan JM. Characteristics of and Important Lessons From the Coronavirus Disease 2019 (COVID-19) Outbreak in China: Summary of a Report of 72314 Cases From the Chinese Center for Disease Control and Prevention. JAMA. 2020. doi: 10.1001/jama.2020.2648.

6. Coronavirus Disease 2019 (COVID-19) Treatment Guidelines [Internet]. NIH; 2020 [citado el 11 de agosto de 2020]. Disponible en: https://www.covid19treatmentguidelines.nih.gov/.

7. Centers for Disease Control and Prevention. Coronavirus Disease 2019 (COVID-19) [Internet]. CDC; 2020 [citado el 11 de agosto de 2020]. Disponible en: https://www.cdc.gov/coronavirus/2019-ncov/ hcp/clinical-guidance-management-patients.html.

8. Clinical management of COVID-19 [Internet]. OMS; 2020 [citado el 11 de agosto de 2020]. Disponible en: https://www.who.int/publications/i/ item/clinical-management-of-covid-19.

9. Bendezu-Quispe G, Rodríguez-Zúñiga MJM, Roman YM, Mori-Llontop LM, Peralta V, Fiestas F. Agentes potencialmente terapéuticos contra el SARS-CoV-2: revisión rápida de la evidencia. Rev Peru Med Exp Salud Pública. 2020;37(2):320-6. doi: 10.17843/rpmesp.2020.372.5409.

10. Dirección General de Medicamentos, Insumos y Drogas. Consumo de dióxido de cloro o clorito de sodio es peligroso para la salud [Internet]. DIGEMID; 2020 [citado el 11 de agosto de 2020]. Disponible en: http://www.digemid.minsa.gob.pe/main.asp?Seccion=3\&IdItem $=2215$.

11. Chuquillanqui F. Coronavirus en Perú [Internet]. RPP noticias; 2020 [citado el 11 de agosto de 2020]. Disponible en: https://rpp.pe/lima/ actualidad/coronavirus-en-peru-dioxido-de-cloro-es-vendido-ilegalmente-a-traves-de-redes-sociales-como-cura-de-la-covid-19-noticia-1282416.

12. Reporte Breve $\mathrm{N}^{\circ} 34$. Uso de dióxido de cloro para el tratamiento de pacientes con diagnóstico de COVID-19 [Internet]. EsSalud: IETSI; 2020 [citado el 11 de agosto de 2020]. Disponible en: http://www. essalud.gob.pe/ietsi/pdfs/covid_19/RB34_dioxidodecloro_19Julio_editado.pdf.

13. Agencia para Sustancias Tóxicas y el Registro de Enfermedades. Resúmenes de Salud Pública. Dióxido de cloro y clorito (Chlorine Dioxide and Chlorite) [Internet]. ATSDR; 2019 [citado el 11 de agosto de 2020]. Disponible en: https://www.atsdr.cdc.gov/es/phs/es_phs160.html.

14. Chlorine Dioxide, Chlorite and Chlorate in Drinking-water [Internet]. OMS; 2016 [citado el 11 de agosto de 2020]. Disponible en: https:// www.who.int/water_sanitation_health/water-quality/guidelines/ chemicals/chlorine-dioxide-chlorite-chlorate-background-jan17.pdf.

15. Kály-Kullai K, Wittmann M, Noszticzius Z, Rosivall L. Can chlorine dioxide prevent the spreading of coronavirus or other viral infections? Medical hypotheses. Physiol Int. 2020;107(1):1-11. doi: $10.1556 / 2060.2020 .00015$.

16. Liberati A, Altman DG, Tetzlaff J, Mulrow C, Gøtzsche PC, Ioannidis JPA, et al. The PRISMA statement for reporting systematic reviews and meta-analyses of studies that evaluate health care interventions: explanation and elaboration. PLoS Med. 2009;6(7):e1000100. doi: 10.1371/journal.pmed.1000100.

17. Higgins JPT, Thomas J, Chandler J, Cumpston M, Li T, Page MJ, Welch VA (editors). Cochrane Handbook for Systematic Reviews of Interventions version 6.0 (updated July 2019) [Internet]. Cochrane; 2019 [citado el 6 de agosto de 2020]. Disponible en: www.training. cochrane.org/handbook.

18. Bramer WM, Giustini D, de Jonge GB, Holland L, Bekhuis T. De-duplication of database search results for systematic reviews in
EndNote. J Med Libr Assoc. 2016;104(3):240-3. doi: 10.3163/15365050.104.3.014.

19. Ouzzani M, Hammady H, Fedorowicz Z, Elmagarmid A. Rayyan-a web and mobile app for systematic reviews. Syst Rev. 2016;5(1):210. doi: 10.1186/s13643-016-0384-4.

20. Determination of the Effectiveness of Oral Chlorine Dioxide in the Treatment of COVID 19 [Internet]. NIH; 2020 [citado el 6 de agosto de 2020]. Disponible en: https://clinicaltrials.gov/ct2/show/ NCT04343742.

21. Eficacia y seguridad del dióxido de cloro para el tratamiento de COVID-19 [Internet]. INS; 2020 [citado el 11 de agosto de 2020]. Disponible en: https://web.ins.gob.pe/sites/default/files/Archivos/ authenticated $\% 2 \mathrm{C} \% 20 \mathrm{administrator} \% 2 \mathrm{C} \% 20$ editor/publicaciones/2020-08-04/SE_24_dioxido\%20de\%20cloro.pdf.

22. Office of the Commissioner. Peligro: No beba la solución mineral milagrosa o productos similares [Internet]. FDA; 2019 [citado el 11 de agosto de 2020]. Disponible en: https://www.fda.gov/consumers/ articulos-en-espanol/peligro-no-beba-la-solucion-mineral-milagrosa-o-productos-similares.

23. European Chemicals Agency. Stopping products sidestepping enforcement through rebranding [Internet]. ECHA; 2019 [citado el 11 de agosto de 2020]. Disponible en: https://newsletter.echa.europa.eu/ home/-/newsletter/entry/stopping-products-sidestepping-enforcement-through-rebranding.

24. Office of the Commissioner. Coronavirus (COVID-19) Update: FDA Warns Seller Marketing Dangerous Chlorine Dioxide Products that Claim to Treat or Prevent COVID-19 [Internet]. FDA; 2020 [citado el 11 de agosto de 2020]. Disponible en: https://www.fda. gov/news-events/press-announcements/coronavirus-covid-19-update-fda-warns-seller-marketing-dangerous-chlorine-dioxide-products-claim.

25. Miracle Mineral Solution and Sodium chlorite solutions [Internet]. Food Standards Agency; 2017 [citado el 11 de agosto de 2020]. Disponible en: https://www.food.gov.uk/business-guidance/miracle-mineral-solution-and-sodium-chlorite-solutions.

26. Organización Panamericana de la Salud. La OPS no recomienda tomar productos que contengan dióxido de cloro, clorito de sodio, hipoclorito de sodio o derivados, 16 de julio del 2020 [Internet]. OPS; 2020 [citado el 11 de agosto de 2020]. Disponible en: https:// iris.paho.org/handle/10665.2/52484.

27. Hagiwara Y, Inoue N. First case of methemoglobinemia caused by a ClO2-based household product. Pediatr Int. 2015;57(6):1182-1183. doi: 10.1111/ped.12708.

28. Chapter 8 Chemical Oxidation and Reduction. In: Studies in Environmental Science. Elsevier; 1979. p. 97-113.

29. Aieta EM, Marco Aieta E, Berg JD. A Review of Chlorine Dioxide in Drinking Water Treatment. American Water Works Association. 1986;78:62-72. doi: 10.1002/j.1551-8833.1986.tb05766.x.

30. Couri D, Abdel-Rahman MS, Bull RJ. Toxicological effects of chlorine dioxide, chlorite and chlorate. Environ Health Perspect. 1982;46:137. doi: 10.1289/ehp.824613.

31. Centers for Disease Control and Prevention. Coronavirus Disease 2019 (COVID-19) - Transmission [Internet]. CDC; 2020 [citado el 11 de agosto de 2020]. Disponible en: https://www.cdc.gov/coronavirus/2019-ncov/prevent-getting-sick/how-covid-spreads.html. 